\title{
Distributed and Decentralized Control of Residential Energy Systems Incorporating Battery Storage
}

\author{
Karl Worthmann, Christopher M. Kellett, Philipp Braun, Lars Grüne, and Steven R. Weller
}

\begin{abstract}
The recent rapid uptake of residential solar photovoltaic (PV) installations provides many challenges for electricity distribution networks designed for one-way power flow from the distribution company to the residential customer. For gridconnected installations, intermittent generation as well as large amounts of generation during low load periods can lead to a degradation of power quality and even outages due to overvoltage conditions. In this paper we present four control methodologies to mitigate these difficulties using small-scale distributed battery storage. These four approaches represent three different control architectures: centralized, decentralized, and distributed control. These approaches are validated and compared using data on load and generation profiles from customers in an Australian electricity distribution network.
\end{abstract}

\section{INTRODUCTION}

Recent years have seen dramatic worldwide growth in small-scale rooftop solar photovoltaic (PV) distributed generation. Over $70 \%$ of the $70 \mathrm{GW}$ installed PV capacity in the European Union (EU) as of 2012, for example, was rooftopmounted (both residential and commerical/industrial) [3].

As PV penetration levels increase, integrating solar PV into the grid creates problems for utilities and customers alike. Reverse power flow in the low-voltage network during daytime periods of peak generation coupled with low residential load leads to well-recognized increases in distribution feeder voltages (the so-called voltage rise problem), with the potential for adverse impacts on power quality and the safety of customerowned devices [9].

In response to these challenges, distributed battery storage is increasingly being considered by utilities seeking to reinforce distribution networks and shave peak demand without largescale capital costs for feeder replacement and related network upgrades [12], [13]. Likewise consumers seeking reduced electricity costs by shifting electricity purchases away from times of peak tariffs, together with a desire for increased energy self-sufficiency, are beginning to consider residential battery storage as a viable option.

While the high capital cost of battery systems has made deployment of residential energy storage systems largely uneconomic, this situation is set to change in the forseeable future through a confluence of falling battery costs, steadily

K. Worthmann is with the Mathematisches Institut, Technische Universität Ilmenau, 99693 Ilmenau, Germany, e-mail: karl.worthmann@tu-ilmenau.de.

C. M. Kellett and S. R. Weller are with the School of Electrical Engineering and Computer Science at the University of Newcastle, Callaghan, New South Wales 2308, Australia, e-mail: \{chris.kellett, steven.weller\} @ newcastle.edu.au.

P. Braun and L. Grüne are with the Mathematisches Institut, Universität Bayreuth, 95440 Bayreuth, Germany, e-mail: \{philipp.braun, lars.gruene\} @uni-bayreuth.de. rising electricity costs, and the opportunity to employ retired electric vehicle batteries in residential storage applications, over and above the storage offered by in-service battery electric vehicles (BEVs) and plug-in hybrid electric vehicles (PHEVs) themselves [16].

With economically viable residential storage on the horizon, researchers have in recent years moved from the analysis of relatively rudimentary and largely uncoordinated battery energy storage systems [11] to systems of increasing scale and sophistication [8], [9], [18], [7], [14].

In this paper, we consider a network of residential energy systems (RESs) where as shown in Figure 1 each RES consists of solar PV generation, battery storage and an inelastic energy load. Each RES is connected to a grid managed by a distribution utility. Four algorithms for controlling battery usage are proposed in this paper, each with the aim of reducing variability in the power demand from the electricity network.

Three of these approaches rely on predictions of load and generation as well as the simple model of an RES to solve an economic Model Predictive Control (MPC) problem. These three economic MPC problems effectively represent three different control architectures: centralized, decentralized, and distributed control. The fourth approach, the Simple Controller, is rule-based, implemented locally and makes no use of a model or predictions of load or generation and hence is a decentralized controller.

Central to the novel distributed control structure proposed in this paper is the idea of a Market Maker (MM), a concept whose roots lie in financial markets; see [4], [2], [17]. In this paper, the MM implements a simple iterative strategy to set prices for buying and selling electricity within a residential network with a view to minimizing the aggregate impact of the $\mathcal{I}$ RESs on the distribution grid. To enable the operation of the MM it is assumed that each RES can communicate with the MM (e.g. via a smart meter), but that the RESs do not communicate directly with one another.

The rest of the paper is organized as follows: in Section II we formalize the Residential Energy System. In Section III we present the four control methodologies. In Section V we apply these algorithms to data from an Australian electricity distribution company to investigate the behavior of the proposed algorithms when applied to a real-world setting. We conclude in Section VI.

\section{The Residential EnERgy System}

We consider a small, neighborhood-level, electricity network consisting of several residences. Each residence com- 


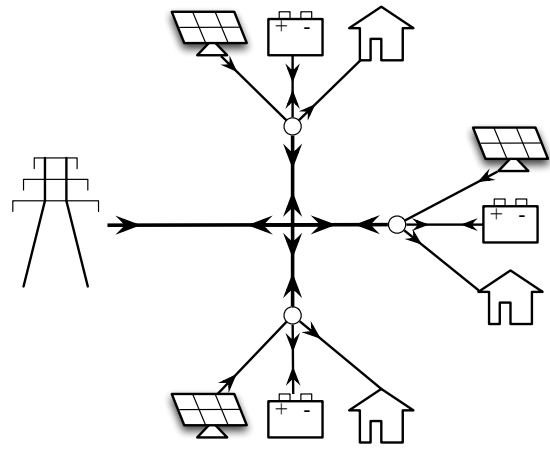

Fig. 1. System model where possible power transfer is indicated by an arrow above. In particular, solar panels only provide power, residential loads only draw power, and the batteries and network can both supply and draw power.

prises a Residential Energy System (RES) as shown in Figure 1, consisting of a residential load, a battery, and solar photovoltaic panels. Each RES is connected to the wider electricity network. Note that, in what follows, the solar photovoltaic panels could be replaced by any residential-scale local generation and the battery could be replaced by any residential-scale local energy storage. The important characteristics of these elements are that the generation and the load are not controllable.

The RES is defined by the following discrete-time system

$$
\begin{aligned}
x(k+1) & =f(x(k), u(k)), \\
y(k) & =h(u(k), w(k))
\end{aligned}
$$

where $x, u, w \in \mathbb{R}^{\mathcal{I}}$ and $\mathcal{I} \in \mathbb{N}$ is the number of RESs connected in the local area under consideration. For user $i, x_{i}$ is the state of charge of the battery in $\mathrm{kWh}, u_{i}$ is the battery charge/discharge rate in $\mathrm{kW}, w_{i}$ is the residential load minus the local generation in $\mathrm{kW}$, and $y_{i}$ is the power supplied by/to the grid in $\mathrm{kW}$. A simple model of the RES of user $i$ is:

$$
\begin{aligned}
x_{i}(k+1) & =x_{i}(k)+T u_{i}(k), \\
y_{i}(k) & =w_{i}(k)+u_{i}(k) .
\end{aligned}
$$

Here, $T$ represents the length of the sampling interval in hours; e.g., $T=0.5$ corresponds to 30 minutes. The state of charge of the battery and the charge/discharge rates of the battery are constrained in practice. In other words, there exist $C_{i}, \bar{u}_{i} \in$ $\mathbb{R}_{>0}$ and $\underline{u}_{i} \in \mathbb{R}_{<0}$, with the units of $C_{i}$ in $\mathrm{kWh}$ and the units of $\bar{u}_{i}, \underline{u}_{i}$ in $\mathrm{kW}$, so that for each RESs $i, i \in\{1, \ldots, \mathcal{I}\}$ :

$$
0 \leq x_{i}(k) \leq C_{i} \quad \forall k \in \mathbb{N}_{0}
$$

and

$$
\underline{u}_{i} \leq u_{i}(k) \leq \bar{u}_{i} \quad \forall k \in \mathbb{N}_{0}
$$

\section{CONTROL ApProACHES}

We present four algorithms for the control of a network of RESs. The first approach, which we term the Simple Controller, is a straightforward rule-based approach for each RES to independently decide when to charge or discharge its battery. The second approach is via a Centralized Model Predictive Control (MPC) algorithm. This scheme requires full communication of all relevant variables for the entire network as well as a known model of the network. As such, this approach is not scalable and, in the simulations that follow, is used as a benchmark for the best possible performance. The third approach is a Decentralized MPC approach that blends the previous two approaches by allowing each RES to implement its own local MPC controller. This requires no communication or cooperation between RESs, similar to the Simple Controller, and implements a receding horizon optimal controller, similar to the Centralized MPC approach. Finally, the fourth approach is a novel Distributed MPC scheme that allows for cooperation amongst the RESs without requiring the full communication overhead or the detailed model of the Centralized MPC approach.

\section{A. Simple Controller}

A simple approach to using a battery in an RES is as follows: If generation exceeds load, and if the battery is not fully charged, then charge the battery. If load exceeds generation, and if the battery is not fully discharged, then discharge the battery (see, e.g., [13]). In a scenario where power can be sold by a residence to the grid, this behavior can be enforced by setting the price for buying power (slightly) higher than the price for selling power.

\section{B. Centralized Model Predictive Control - Benchmark Perfor- mance}

For the Simple Controller proposed in the previous section, if the load always exceeds generation then the battery will never be used (see the response of RES 3 in Figure 2 below). However, a battery can be used to time-shift energy consumption and, hence, can be used to flatten the usage profile. In order to achieve the goal of reducing the aggregate variation in energy usage across the network, a Centralized MPC scheme is presented. Here, a central entity makes all decisions based on unlimited information exchange between the subsystems.

MPC is a control strategy that aims to improve system behavior by iteratively minimizing an optimization criterion with respect to predicted trajectories and implementing the first part of the resulting optimal control sequence until the next optimization is performed (see, e.g., [10], [15] or [6] for details). We propose such a predictive controller for (3). In order to do this, we assume that we have predictions of the residential load and generation some time into the future that is coincident with the horizon of the predictive controller. In other words, given a prediction horizon $N \in \mathbb{N}$, we assume knowledge of $w_{i}(j)$ for all $j \in\{k, \ldots, k+N-1\}$, where $k \in \mathbb{N}_{0}$ is the current time. When the prediction horizon is less than a day, i.e. $N T \leq 24$, such an assumption is not initially unreasonable as residential loads tend to follow daily patterns and one-day ahead weather predictions can be fairly accurate.

Define the predicted average power usage for the $i^{\text {th }}$ RES as

$$
\zeta_{i}(k):=\frac{1}{N} \sum_{j=k}^{k+N-1} w_{i}(j)
$$


To implement a Centralized MPC controller, we then compute the overall average on the considered prediction horizon by

$$
\bar{\zeta}(k):=\frac{1}{\mathcal{I}} \sum_{i=1}^{\mathcal{I}} \zeta_{i}(k)
$$

and then minimize the joint cost function

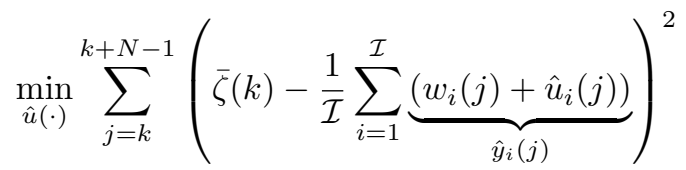

with respect to $\hat{u}(k), \hat{u}(k+1), \ldots, \hat{u}(k+N-1)$ with $\hat{u}(j)=$ $\left(\hat{u}_{1}(j), \hat{u}_{2}(j), \ldots, \hat{u}_{\mathcal{I}}(j)\right)^{T}, j=k, k+1, \ldots, k+N-1$, subject to the system dynamics (3), the current state $x(k)=$ $\left(x_{1}(k), \ldots, x_{\mathcal{I}}(k)\right)^{T}$, and the battery constraints (4)-(5) for all $i \in\{1, \ldots, \mathcal{I}\}$. Here, and in what follows, we denote predicted controls, states, and outputs in the MPC algorithm by hats; i.e., for the $i^{\text {th }}$ RES at time $j$ the predicted control is $\hat{u}_{i}(j)$, predicted battery state of charge is $\hat{x}_{i}(j)$, and the predicted output is $\hat{y}_{i}(j)$.

Remark 3.1: In the classical MPC approach one assumes the existence of an optimal steady-state solution and then the task of the MPC feedback controller is to stabilize this steadystate operating point. This is reflected in the cost function that is minimized in the MPC problem where the cost function will attain a minimum at the steady-state operating point. For several reasons, this is not always desirable and has led to the recent introduction of economic MPC [1] where the defining characteristic of an economic MPC problem is either the lack of an optimal steady-state solution or that the chosen cost function fails to attain a minimum at the steady-state solution. On the basis of the form of our chosen cost function (7), we are proposing an economic MPC approach without terminal constraints [5].

\section{Decentralized Model Predictive Control}

The Centralized MPC approach presented above requires a significant amount of communication overhead since each RES has to communicate its predicted usage profile to a central entity, which then needs to send an individual control sequence (in the form of a battery usage profile) to each RES. Additionally, as the size of the network grows, the computation time required to solve the optimization problem becomes very large. A further drawback of the Centralized MPC approach is that the central entity requires full knowledge of the network model, in particular (4)-(5) for each $i \in\{1, \ldots, \mathcal{I}\}$. Therefore, any change in the network such as the addition of new solar resources or batteries, requires the central entity to update its model. As a consequence of these limitations, it is of interest to design decentralized or distributed control approaches that alleviate the communication and computation difficulties, as well as the need for an up-to-date centrally maintained model of the network, encountered in Centralized MPC.

A straightforward option in order to flatten the energy profile of the $i^{\text {th }}$ RES is to penalize deviations from its (anticipated) average usage defined in (6). With a quadratic cost function, this leads to the finite-horizon optimal control problem

$$
\min _{\hat{u}_{i}(\cdot)} \sum_{j=k}^{k+N-1}(\underbrace{w_{i}(j)+\hat{u}_{i}(j)}_{\hat{y}_{i}(j)}-\zeta_{i}(k))^{2}
$$

subject to the system dynamics (3), the current battery state of charge $x_{i}(k)$, and the battery constraints (4)-(5).

This optimization is performed by each RES individually with no reference to the rest of the network. This removes the aforementioned communication and computation difficulties of Centralized MPC since no communication is required and the optimization problem for the $i^{\text {th }}$ RES is limited to include only its own operation. However, it is well-known that such a decentralized approach is unlikely to lead to, or even approach, network-wide optimal behavior. In order to improve networkwide behavior, we next introduce a Distributed MPC approach that makes use of some communication within the network but keeps the optimal control problems local to each RES.

\section{Market Maker Distributed MPC}

We propose a novel hierarchical distributed control approach where each RES can communicate with a centralized entity, called the Market Maker (MM) (see [4]), with the aim of achieving some network-wide objective. The objective we pursue in this work is to flatten the aggregate power usage of the network.

In what follows the price of buying or selling power from or to the grid, respectively, for an RES is discussed. It is important in this context to note that these need not be monetary prices, but, rather, can be viewed as a mechanism to enforce reasonable cooperation between RESs within the network.

Denote the price for buying power from the grid by $p$ : $\mathbb{N}_{0} \rightarrow \mathbb{R}_{\geq 0}$ and the price to sell power to the grid by $q$ : $\mathbb{N}_{0} \rightarrow \mathbb{R}_{\geq 0}$. In many current electricity markets the values of $p$ and $q$ are constant; i.e., are independent of the time index $k$. In markets that implement time-of-use pricing, the $p$ and $q$ are periodic with a period of 24 hours, with higher values at times of predicted high usage and lower values at times of predicted low usage. In the sequel, these prices will be manipulated by the Market Maker in real-time in order to obtain desirable behavior from the residential network. By a slight abuse of notation, we will refer to the length $N$ sequence of prices from time $k$ as $\mathbf{p}=(p(k), \ldots, p(k+N-1))^{T}$ and $\mathbf{q}=(q(k), \ldots, q(k+N-1))^{T}$. Define the quantities

$$
y_{i}^{+}(k):=\max \left\{y_{i}(k), 0\right\}, \text { and } y_{i}^{-}(k):=\max \left\{-y_{i}(k), 0\right\}
$$

so that $y^{+}(k)$ is the power drawn from the grid at time $k$ while $y^{-}(k)$ is the power supplied to the grid at time $k$. We observe that only one of $y^{+}(k)$ or $y^{-}(k)$ can be nonzero at each $k$. We denote the $N \times N$ identity matrix by $I_{N}$, the $N$ vector $(1,1, \ldots, 1)^{T}$ by $\mathbb{1}_{N}$, the $N \times N$ matrix of all zeros by $\mathbf{0}_{N}$, and the $N \times N$ lower triangular matrix consisting of ones and zeros by

$$
L_{N}=\left[\begin{array}{ccc}
1 & & 0 \\
\vdots & \ddots & \\
1 & \cdots & 1
\end{array}\right]
$$


We define the finite-horizon optimal control problem for each RES so as to minimize the cost of an individual residence over an $N$-step horizon; i.e., for subsystem $i, i \in$ $\{1,2, \ldots, \mathcal{I}\}$,

$$
\min _{\hat{u}_{i}(\cdot)} \underbrace{\sum_{j=k}^{k+N-1} p(j) \hat{y}_{i}^{+}(j)-q(j) \hat{y}_{i}^{-}(j)}_{=: 0^{T} \hat{u}_{i}+\mathbf{p}^{T} \hat{y}_{i}^{+}-\mathbf{q}^{T} \hat{y}_{i}^{-}}
$$

subject to the constraints (4) representing the battery capacities and system dynamics (3); i.e. define $\widetilde{w}_{i}(k) \in \mathbb{R}^{N}$ by $\widetilde{w}_{i}(k):=$ $\left[w_{i}(k), w_{i}(k+1), \ldots, w_{i}(k+N-1)\right]^{T}$. Then

$$
\left[\begin{array}{rrr}
T \cdot L_{N} & \mathbf{0}_{N} & \mathbf{0}_{N} \\
-T \cdot L_{N} & \mathbf{0}_{N} & \mathbf{0}_{N} \\
-I_{N} & I_{N} & -I_{N} \\
I_{N} & -I_{N} & I_{N}
\end{array}\right]\left(\begin{array}{c}
\hat{u}_{i} \\
\hat{y}_{i}^{+} \\
\hat{y}_{i}^{-}
\end{array}\right) \leq\left(\begin{array}{c}
\frac{\left(C-x_{i}(k)\right) \cdot \mathbb{1}_{N}}{x_{i}(k) \cdot \mathbb{1}_{N}} \\
\frac{\widetilde{w}_{i}(k)}{-\widetilde{w}_{i}(k)}
\end{array}\right)
$$

as well as the constraints (5); i.e., $\underline{u} \cdot \mathbb{1}_{N} \leq \hat{u}_{i} \leq \bar{u} \cdot \mathbb{1}_{N}$. Here, the optimization variables are

$$
\begin{aligned}
\hat{u}_{i} & =\left(\hat{u}_{i}(k), \ldots, \hat{u}_{i}(k+N-1)\right)^{T}, \\
\hat{y}_{i}^{+} & =\left(\hat{y}_{i}^{+}(k), \ldots, \hat{y}_{i}^{+}(k+N-1)\right)^{T}, \\
\hat{y}_{i}^{-} & =\left(\hat{y}_{i}^{-}(k), \ldots, \hat{y}_{i}^{-}(k+N-1)\right)^{T} .
\end{aligned}
$$

Since this is a linear optimization problem, the optimum is attained in each minimization - although it may not be unique. Note that the minimization problem to be solved encompasses $3 N$ variables and $6 N$ constraints yielding linear growth of the number of optimization variables and constraints in the prediction horizon. Furthermore, it can be observed that any solution necessarily exhibits $\hat{x}_{i}(k+N-1)=0$. This is intuitively obvious since there is no benefit to having a charge left in the battery at the end of the horizon. However, this does not necessarily imply that the $i^{\text {th }}$ component of the closed loop solution at time $k+N-1$ equals zero due to the receding horizon nature of MPC.

In order to set prices, we propose an iterative negotiation before prices are set. This negotiation is operated by the Market Maker, which sets initial prices from the current time $k$ to the end of the prediction horizon $k+N-1$ and broadcasts these to the residential network. Each RES then solves its own MPC problem based on the cost functional given by (8), then communicates its desired grid profile, $\left\{\hat{y}_{i}(j)\right\}_{j=k}^{k+N-1}$, to the Market Maker. The Market Maker uses the aggregated grid profile to update prices, which are then broadcast to the residential network. This process is iterated until (hopefully) a steady-state is reached. Note that in a slight modification to the original definition of a Market Maker proposed in [4] we allow multiple iterations of the Market Maker setting prices and receiving bids whereas in [4] the Market Maker receives bids once and sets prices once (i.e., there is only a single iteration per sampling instant). Herein we propose a simple algorithm for the setting of prices by the Market Maker. However many algorithms are possible (see, e.g., [17]) and investigating these alternatives is the subject of ongoing work.

Denote the negotiation iteration index by $\ell \in \mathbb{N}_{0}$ and the predicted grid profile for RES $i, i \in\{1, \ldots, \mathcal{I}\}$, at negotiation iteration $\ell$ by $\left\{\hat{y}_{i, \ell}(j)\right\}_{j=k}^{k+N-1}$. Denote the predicted demand of the residential network at time $j$ and negotiation iteration $\ell$ by

$$
\Pi_{\ell}(j):=\frac{1}{\mathcal{I}} \sum_{i=1}^{\mathcal{I}} \hat{y}_{i, \ell}(j) \quad \forall j \in\{k, \ldots, k+N-1\}
$$

and the average predicted demand as

$$
\bar{\Pi}(j):=\frac{1}{N} \sum_{j=k}^{k+N-1} \Pi_{\ell}(j) .
$$

Remark 3.2: Note that since the predicted residential load and generation is fixed for the prediction horizon, the average predicted demand, $\bar{\Pi}(j)$, is independent of the iterative negotiation process.

Let $p, \bar{p} \in \mathbb{R}_{\geq 0}$ be the minimum and maximum buying prices, respectively. The buying price $p_{\ell+1}(j)$ of the successor iteration is set by the Market Maker for each $j \in\{k, \ldots, k+$ $N-1\}$ as

$$
p_{\ell+1}(j)=\max \left\{\underline{p}, \min \left\{\bar{p}, p_{\ell}(j)+\theta\left(\Pi_{\ell}(j)-\bar{\Pi}(j)\right)\right\}\right\}
$$

where $\theta \in \mathbb{R}_{>0}$ is a selectable parameter. The selling price $q_{\ell+1}(j)$ is set to a fraction of $p_{\ell+1}(j)$; i.e. $q_{\ell+1}(j)=\kappa p_{\ell+1}(j)$, $\kappa \in(0,1)$. This convention ensures that certain pathological arbitrage-type behavior is avoided since $p_{\ell}(j) \geq q_{\ell}(j)$ for all $\ell$ and $j$. In other words, buying electricity at a particular time is always more expensive than what can be obtained by selling electricity at that time. This inhibits an RES shortselling electricity; i.e., buying a lot of power to charge its battery at one time instant and then turning around and selling that power at the next time instant at a profit. Note that in the presence of realistic charging/discharging rate constraints this may not be a problem.

In the simulations of the following sections we set $\theta=0.2$, $\kappa=0.95, \underline{p}=0$, and $\bar{p}=5$. The initial prices are set as $p_{0}(j)=\max \left\{\underline{p}, \min \left\{\bar{p}, \frac{1}{\mathcal{I}} \sum_{i=1}^{\mathcal{I}} \omega_{i}(j)-\bar{\Pi}(j)\right\}\right\}$. Several different values were tried for the number of Market Maker iterations and values between 3 and 10 provided the best performance. Consequently, the results reported below use 3 Market Maker iterations; i.e., $\ell=0,1,2,3$. Understanding the convergence behavior of the iterative Market Maker is an important element of future work.

Summarizing, the Market Maker increases both the selling and buying price at time $j$ when network demand at time $j$ exceeds the average predicted demand and, conversely, if the network demand is less than the average predicted demand the Market Maker decreases the prices. Intuitively, this should have the effect of flattening the aggregate power drawn or supplied from the residential energy network. In the sequel, we will refer to the scheme just described as Market Maker Distributed MPC (MM Distributed MPC).

\section{Synthetic Trajectory Simulations}

To facilitate our discussion of the four algorithms presented above for control of RESs we will make use of the three synthetic power profiles $w_{i}$ depicted in Figure 2 representing three synthetic RESs. In Section V we will apply the four algorithms to data from an Australian electricity network. 


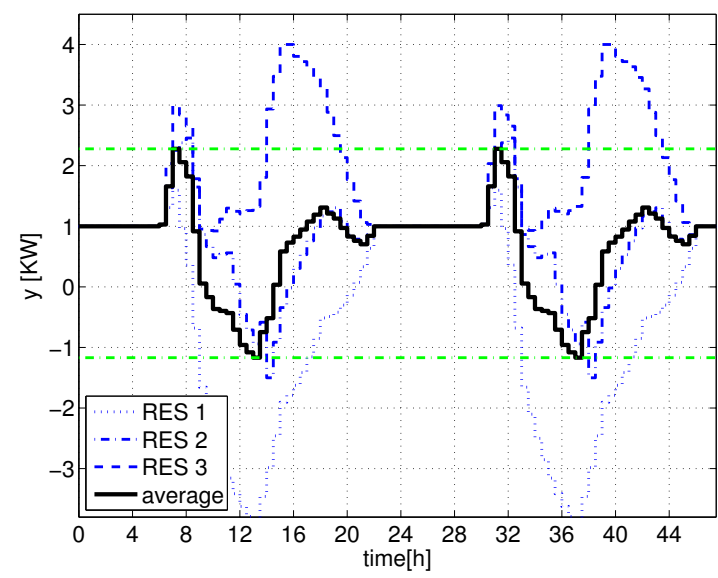

Fig. 2. Power consumption without use of battery storage (no power management controller) is shown for 48 hours. The average consumption peaks are indicated by the dash dotted green lines.

Throughout this section, we fix the parameters $x_{i}(0)=0.5$, $C_{i}=4, \underline{u}_{i}=-0.5$, and $\bar{u}_{i}=0.5$. It is not necessary in general for the storage capacities of the individual subsystems to be equal. We have imposed this constraint in order to simplify the presentation of our numerical findings.

With the stated goal of flattening the power demand profile we will compare the different proposed controllers against each other using two metrics. Define the average power demand at time $k$ as

$$
\Pi(k):=\frac{1}{\mathcal{I}} \sum_{i=1}^{\mathcal{I}} y_{i}(k) .
$$

Let $\mathcal{N}$ denote the simulation length in number of samples; e.g. in Figure $2, \mathcal{N}=48 \mathrm{hrs} \times \frac{\text { samples }}{0.5 \mathrm{hrs}}=96$. The first performance metric is the peak-to-peak (PTP) variation of the average demand of all RESs given by

$$
\left(\max _{k \in\{0, \ldots, \mathcal{N}-1\}} \Pi(k)\right)-\left(\min _{k \in\{0, \ldots, \mathcal{N}-1\}} \Pi(k)\right) .
$$

The second performance metric is the root-mean-square (RMS) deviation from the average; i.e., we calculate the average $\Upsilon:=\frac{1}{\mathcal{N} \mathcal{I}} \sum_{k=0}^{\mathcal{N}-1} \sum_{i=1}^{\mathcal{I}} w_{i}(k)$, and the respective quadratically penalized deviations

$$
\sqrt{\frac{1}{\mathcal{N}} \sum_{k=0}^{\mathcal{N}-1}(\Pi(k)-\Upsilon)^{2}} .
$$

Throughout this section we will provide plots showing average power demand as well as battery state of charge. We draw attention to the time window on these plots as being from hour 25 to hour 48 . This shows the results for a 24 hour period where the starting point of hour 25 is shown as the effect of the choice of initial condition has essentially disappeared after one day.

\section{A. The Nominal Case}

We first apply the four controllers proposed in Section III to the three synthetic power profiles of Figure 2 with the resulting average power profiles shown in Figure 3. a) Simple Controller

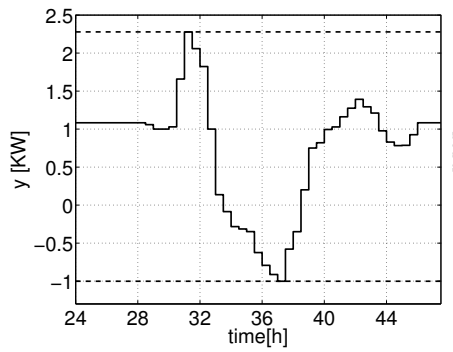

c) Decentralized MPC

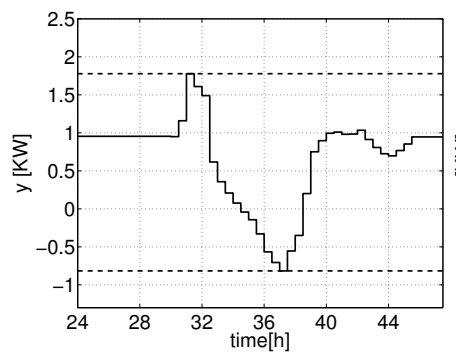

b) Centralized MPC

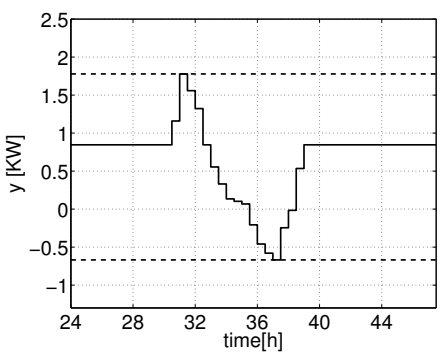

d) MM Distributed MPC

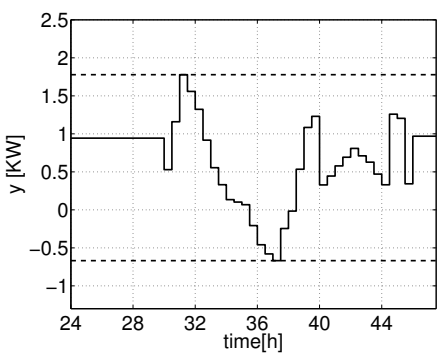

Fig. 3. Average power demand for the controllers proposed in Section III with one day prediction horizon $(N=48, T=0.5)$ : $y_{i}(k), k=24,25, \ldots, 47$.

The peak-to-peak variations and the RMS deviations from the average for the no-storage case as well as the four controllers are collected in Table I.

\begin{tabular}{|r|c|c|c|c|}
\hline & $\begin{array}{c}\text { peak } \\
\text { (high) }\end{array}$ & $\begin{array}{c}\text { peak } \\
\text { (low) }\end{array}$ & $\begin{array}{c}\text { PTP } \\
\text { Variation }\end{array}$ & $\begin{array}{c}\text { RMS } \\
\text { Deviation }\end{array}$ \\
\hline No Battery Storage & 2.2785 & -1.1683 & 3.4468 & 0.7865 \\
\hline Simple Controller & 2.2785 & -1.0016 & 3.2801 & 0.7573 \\
\hline Decentralized MPC & 1.7785 & -0.8168 & 2.5952 & 0.6076 \\
\hline MM Distributed MPC & 1.7785 & -0.6683 & 2.4468 & 0.5399 \\
\hline Centralized MPC & 1.7785 & -0.6683 & 2.4468 & 0.5079 \\
\hline
\end{tabular}

TABLE I

COMPARISON OF PEAK-TO-PEAK VARIATION AND RMS DEVIATION FOR DIFFERENT CONTROL TECHNIQUES APPLIED TO SYNTHETIC TRAJECTORIES OF FIGURE 2.

Since it has access to all available information and minimizes the deviation from the average globally, the Centralized MPC algorithm of Section III-B represents the best possible outcome with respect to flattening the average grid profile and Table I verifies this. In this case we see a reduction of the total variation by $1 \mathrm{~kW}$ can be achieved which corresponds to the theoretical maximum in view of the charging rate constraints $\left(\bar{u}_{i}-\underline{u}_{i}=1\right)$. As noted at the beginning of Section III-C, this performance comes at a significant cost in terms of complexity of the implementation and a high computational effort.

For these trajectories, we see that the MM Distributed MPC algorithm of Section III-D achieves the same peakto-peak variation as the Centralized MPC, but without the requirement of maintaining a global model of all RESs or of needing to solve a large global optimization problem. The Decentralized MPC of Section III-C removes the need for any communication infrastructure but results in slightly worse performance than the MM Distributed MPC algorithm. The Simple Controller of Section III-A results in a negligible 
improvement over the case of having no battery storage.

In order to get a feel for how the different controllers behave, we plot the battery state of charge over time in Figure 4. We observe that for the Simple Controller, RES 3 makes no use of its battery due to the fact that load always exceeds generation (since $y_{3}(k)>0$ for all $k$ in Figure 2). By contrast, when applying Decentralized MPC RES 3 charges its battery in the morning in order to reduce the large afternoon peak. It is interesting to note that the battery usage is effectively the same for all RESs under the MM Distributed MPC approach, while the Centralized MPC approach leads to individual but similar battery usage for each RES.

a) Simple Controller

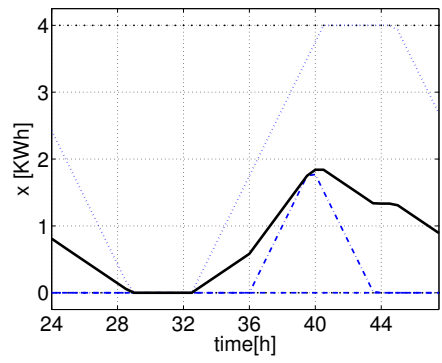

c) Decentralized MPC

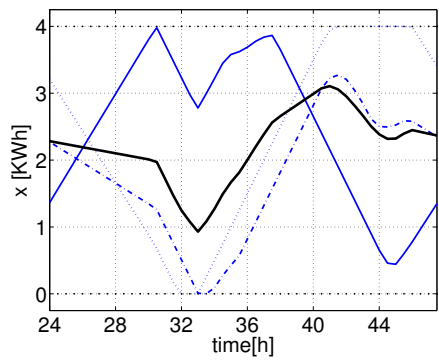

b) Centralized MPC

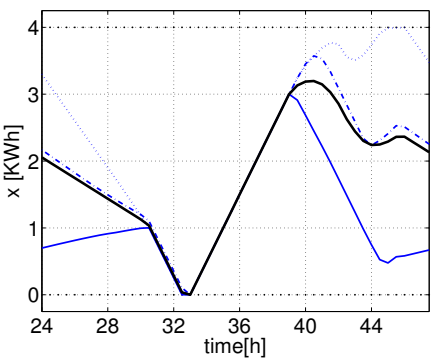

d) MM Distributed MPC

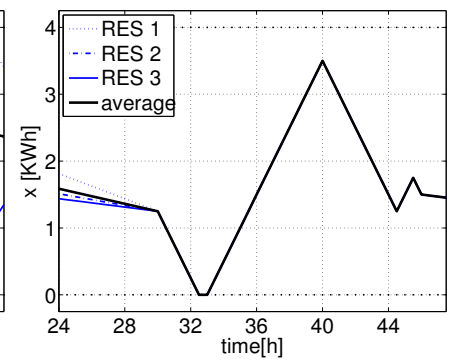

Fig. 4. Battery usage profiles for the four controllers applied to the synthetic trajectories of Figure 2 with a one day prediction horizon $(N=48, T=0.5)$ for the MPC-based controllers: $x_{i}(k), k=24,25, \ldots, 47$.

\section{B. Performance under different Prediction Horizons}

We briefly investigate the change in performance of the three MPC algorithms when we change the length of the prediction horizon. With regards to solar predictions, shorter prediction horizons are more reliable and we here focus on prediction horizons of 24 hours or less.

With respect to the criterion of peak-to-peak variation, the differences between a 6,12 , or 24 hour prediction horizon are almost negligible. At a short prediction horizon of 3 hours, the performance of the MM Distributed MPC and the Centralized MPC algorithms begins to deteriorate. The RMS deviations from the average for the three MPC schemes are shown in Table II. Again, the performance of the MM Distributed MPC is close to the performance of the Centralized MPC, and both outperform the Decentralized MPC algorithm. We observe that there is negligible benefit to a prediction horizon of 24 hours over a prediction horizon of 12 hours. A more comprehensive investigation of the effects of prediction horizon length is left for future work.

\begin{tabular}{|r||c|c|c|c|}
\hline Horizon & $3 \mathrm{~h}$ & $6 \mathrm{~h}$ & $12 \mathrm{~h}$ & $24 \mathrm{~h}$ \\
\hline \hline Decentralized MPC & 0.7057 & 0.6636 & 0.6166 & 0.6076 \\
\hline MM Distributed MPC & 0.6739 & 0.6192 & 0.5393 & 0.5399 \\
\hline Centralized MPC & 0.7112 & 0.6186 & 0.5365 & 0.5079 \\
\hline
\end{tabular}

TABLE II

RMS DEVIATIONS FROM THE AVERAGE FOR THE MPC-BASED CONTROLLERS USING DIFFERENT PREDICTION HORIZONS APPLIED TO THE SYNTHETIC TRAJECTORIES IN FIGURE 2.

\section{Performance Under Inaccurate Forecasts}

The MPC-based controllers rely on the predicted demand data $w_{i}(j), j=k, k+1, \ldots, k+N-1$, which is the difference between the predicted residential load and the predicted solar PV generation. However, both of these predictions are subject to random fluctuations due to a variety of factors, including variability in consumption and in cloud cover. Hence, in this section we add noise to the predictions of the individual subsystems and investigate the sensitivity of the proposed MPC schemes to inaccurate forecasts. To be more precise, $\Theta$ realizations $\left(\xi_{i}^{\rho}(j),(i, j) \in\{1,2, \ldots, \mathcal{I}\} \times\{0,1, \ldots, \mathcal{N}+N-1\}\right)$, $\rho \in\{1,2, \ldots, \Theta\}$, of independently and identically normally distributed random variables with zero mean and standard deviation $0.01 \cdot T$ are generated. Then, for each time instant $k$, each system $i$, and each realization $\rho$, a disturbance sequence $\varrho_{i, k}^{\rho}(\cdot)$ is generated according to

$$
\varrho_{i, k}^{\rho}(j+1)=\varrho_{i, k}^{\rho}(j)+\xi_{i}^{\rho}(k+j), \quad \varrho_{i, k}^{\rho}(0)=0 .
$$

This disturbance sequence captures two important elements of likely forecast errors. The first is that the accuracy of forecasts is degraded for times farther into the future. This is captured by the fact that the standard deviation increases with time. The second element is that disturbances are likely to be correlated in time since weather and load conditions are unlikely to change rapidly over time. This observation is consistent with the real-world data used in Section V.

Based on these disturbance sequences, disturbed forecasts $\widetilde{w}_{i, k}^{\rho}(\cdot)$ are constructed by

$$
\widetilde{w}_{i, k}^{\rho}(\cdot)=w_{i}(k+\cdot)+\varrho_{i, k}^{\rho}(\cdot) .
$$

Based on these disturbed predicted energy demands, a MonteCarlo simulation with $\Theta=1000$ realizations of the stochastic processes $\left(\xi^{\rho}(\cdot)\right), \rho \in\{1,2, \ldots, \Theta\}$ is carried out. We calculate the two performance metrics PTP variation and RMS deviation for each of the $\Theta$ realizations. The extrema as well as the averages are given in Table III. In other words, we present the largest and smallest PTP variation and RMS deviation and also the average of both metrics over all realizations.

\begin{tabular}{|r|c|c|}
\hline & $\begin{array}{c}\text { PTP Variation } \\
\text { Max. / Av. / Min. }\end{array}$ & $\begin{array}{c}\text { RMS Deviation } \\
\text { Max. / Av. / Min. }\end{array}$ \\
\hline Decentralized MPC & $2.7171 / 2.5939 / 2.4913$ & $0.6181 / 0.6040 / 0.5877$ \\
\hline MM Distributed MPC & $2.4951 / 2.4475 / 2.4283$ & $0.6158 / 0.5913 / 0.5691$ \\
\hline Centralized MPC & $2.4880 / 2.4474 / 2.4283$ & $0.5156 / 0.5104 / 0.5062$ \\
\hline
\end{tabular}

TABLE III

COMPARISON OF PEAK-TO-PEAK VARIATION AND RMS DEVIATION FOR DIFFERENT CONTROL TECHNIQUES AND INACCURATE FORECASTS. 
We see that the relative performance ordering is the same as before in all cases: Centralized MPC provides the best performance while MM Distributed MPC sometimes approaches the performance of Centralized MPC and outperforms Decentralized MPC. When compared with the nominal (unperturbed) values from Table I, we observe that none of the MPC-based schemes suffer significant performance degradations when using inaccurate forecasts.

Additionally, in Figure 5 we show the envelopes obtained by taking the pointwise in time maximum and minimum values across all realizations as well as the nominal (unperturbed) trajectories for comparison. Here, large variations contributing to the RMS deviation criterion can be observed for the MM Distributed MPC. These typically occur if the charging rate constraints are not active, i.e., $u_{i}(k) \neq \underline{u}$ and $u_{i}(k) \neq \bar{u}$, $i=1,2, \ldots, \mathcal{I}$, while MM Distributed MPC performs very well during longer (dis-)charging intervals. Hence, future research will aim at improving the MM Distributed MPC in order to alleviate this drawback. Nonetheless, MM Distributed MPC is still competitive in comparison to Decentralized MPC with respect to this metric; indeed it even slightly outperforms it. Consequently, we again see that our proposed modelbased control schemes are not overly sensitive to inaccurate forecasts.

a) Centralized MPC
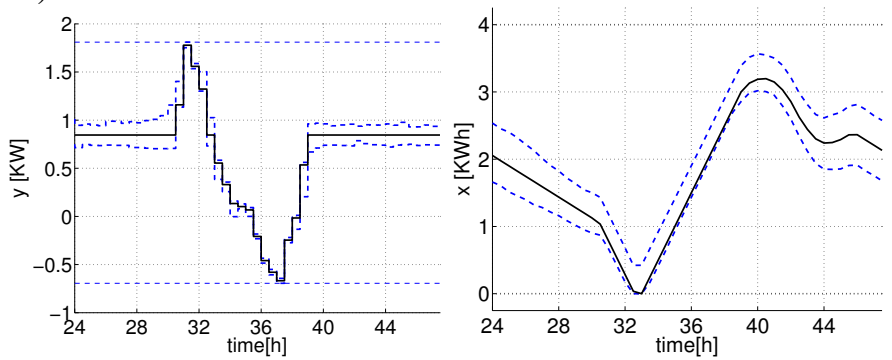

b) Decentralized MPC
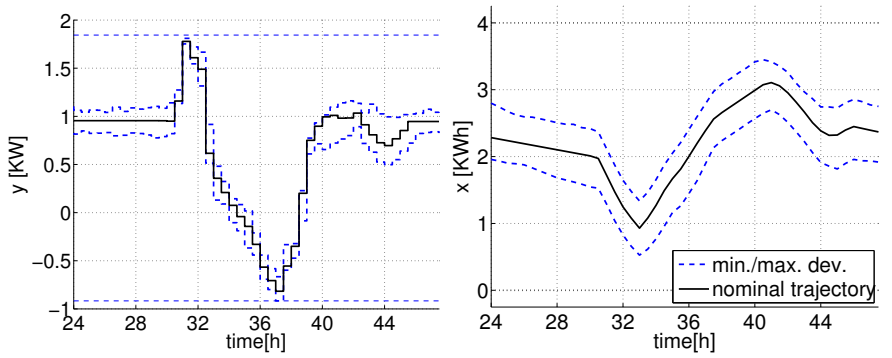

c) MM Distributed MPC
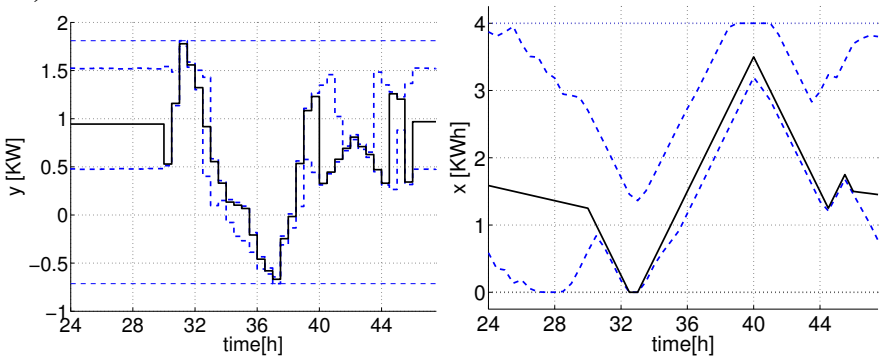

Fig. 5. The impact of inaccurate forecasts on the average power demands (left) and the battery usage profiles (right) when using the MPC-based controllers applied to the synthetic trajectories.

\section{Australian Data}

In this section, we compare the four previously discussed controllers by considering the load and generation profiles for a group of 20 and a group of 300 customers drawn from the Australian electricity distribution company Ausgrid. Ausgrid is a state-owned corporation servicing approximately 1.6 million customers across New South Wales from Sydney to Newcastle. The data from these customers was collected as part of the Smart Grid, Smart City project and covers 12 months, of which we use six weeks starting on the first of March 2011. For the set of 20 (300) customers the mean consumption is +0.4209 $(+0.4282)$ while the maximum and minimum values for the average trajectory are $+1.3740(+1.0375)$ and -0.3414 $(-0.2153)$, respectively. This represents the no battery case.

Applying the controllers proposed in Section III yields the load/generation and battery state of charge profiles shown in Figure 6 with the peak-to-peak variation and the RMS deviation from the average shown in Table IV. Average demand profiles corresponding to 300 systems are not shown as they are similar to the 20 systems case.

\begin{tabular}{|r|c|c|}
\hline & PTP Variation & RMS Deviation \\
\hline No Battery Storage & $1.7153(1.2528)$ & $0.2970(0.2525)$ \\
\hline Simple Controller & $1.6388(1.1470)$ & $0.2848(0.2397)$ \\
\hline Decentralized MPC & $1.2098(0.8402)$ & $0.1952(0.1509)$ \\
\hline MM Distributed MPC & $1.1154(0.8468)$ & $0.1765(0.1447)$ \\
\hline Centralized MPC & 1.1153 (N/A) & $0.1267 \quad$ (N/A) \\
\hline
\end{tabular}

TABLE IV

Australian Data: PEAK-TO-PEAK VARIATION AND RMS DEVIATION FROM THE AVERAGE FOR 20 (300) RESS. THE CORRESPONDING PLOTS ARE SHOWN IN FIGURE 6.

In Table IV, when applied to the group of 20 RESs we observe that the peak-to-peak variation in power demand is significantly reduced by all three MPC-based schemes. Indeed, when considering peak-to-peak variation, both the Decentralized MPC and the MM Distributed MPC yield performance close to the benchmark given by Centralized MPC; with the distributed MPC scheme slightly outperforming the Decentralized MPC scheme. When considering the RMS deviation from the average for 20 RESs we see that the MPC-based controllers provide a significant improvement over no storage and the Simple Controller, while the Centralized MPC scheme provides another significant improvement over the other two MPC-based schemes.

Centralized MPC is not applicable for 300 RESs since the resulting optimization problem becomes too large to be solved in a reasonable period of time. Therefore, we do not have Centralized MPC as a benchmark of performance when considering 300 RESs. In this case, we see that both Decentralized MPC and MM Distributed MPC outperform the Simple Controller in terms of both peak-to-peak variation and RMS deviation from the average. Interestingly, for 300 RESs, the Decentralized MPC scheme slightly outperforms the MM Distributed MPC scheme when considering peak-to-peak variation, while this slight performance difference is reversed when considering RMS deviation from the average. This is a finding that requires further investigation and is left for future work. 
a) Simple Controller
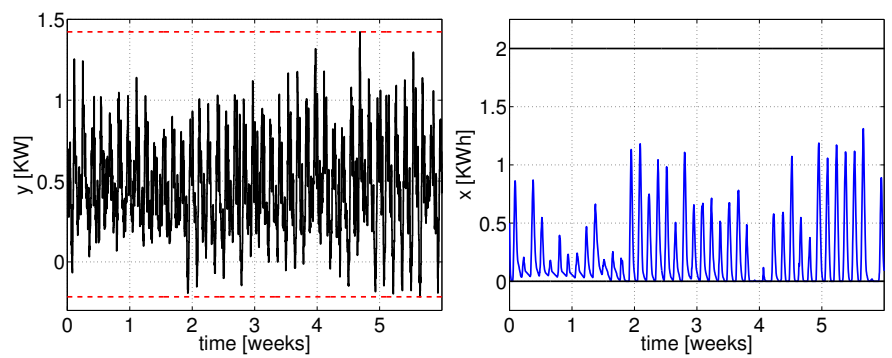

b) Centralized MPC
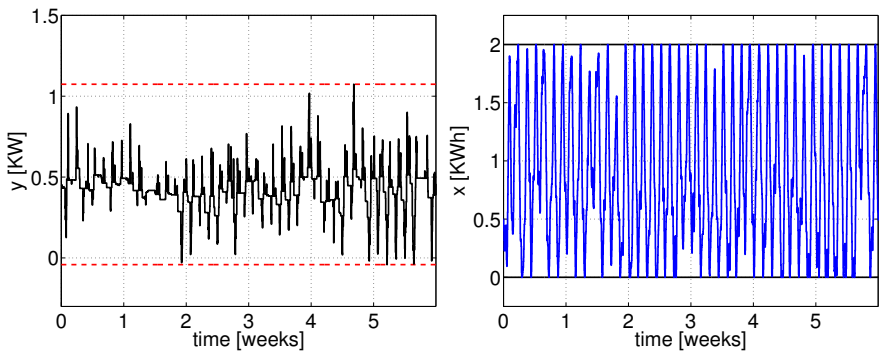

c) Decentralized MPC
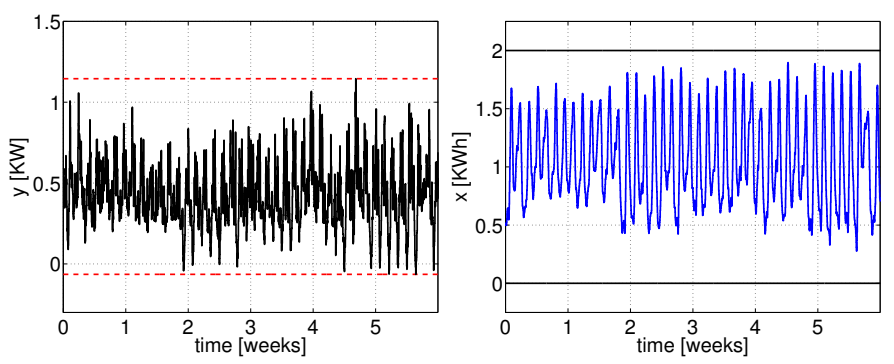

d) MM Distributed MPC
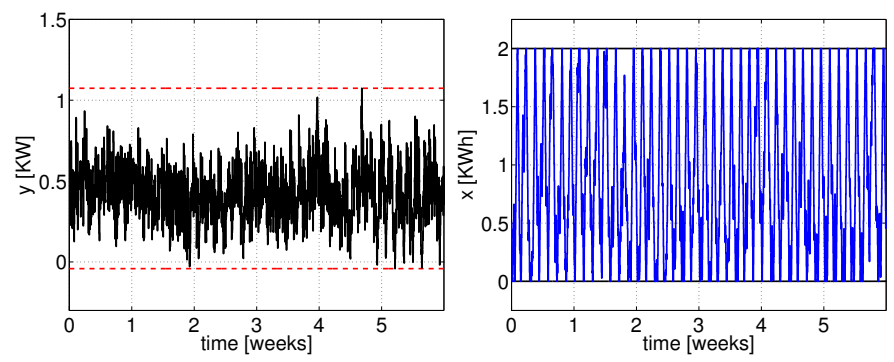

Fig. 6. Australian data for twenty customers: average power demand and battery state of charge profile when applying the four different control schemes over six weeks.

In Figure 6 we observe that the MM Distributed MPC scheme appears to better utilize the available battery storage when compared with the Decentralized MPC scheme in the sense that for the decentralized scheme the batteries are on average never empty or full. By contrast, the MM Distributed MPC scheme fully employs the available battery capacity in order to further flatten the aggregate grid profile. This observation also holds true for the scenario with 300 RESs.

\section{CONCLUSIONS}

Of the four algorithms presented in this paper, Centralized MPC provides the best performance but quickly becomes infeasible as the size of the required optimization problem rapidly increases with the network size. Decentralized MPC has the benefit of requiring no communication between RESs (or any central entity) while ensuring that the size of the local optimization problems remains small. However, in general it is known that such decentralized schemes do not lead to network-wide optimal behavior. The novel Distributed MPC scheme proposed in this paper requires a central entity (as in Centralized MPC), but does not suffer from scalability problems since the optimization problems remain local to each RES.

\section{ACKNOWLEDGEMENT}

The assistance of E. Ratnam in facilitating access to the Ausgrid data is gratefully acknowledged.

\section{REFERENCES}

[1] D. Angeli, R. Amrit, and J. B. Rawlings. On average performance and stability of economic model predictive control. IEEE Transactions on Automatic Control, 57(7):1615-1626, July 2012.

[2] A. Beja and M. B. Goldman. On the dynamic behavior of prices in disequilibrium. J. Financ., 35(2):235-248, 1980.

[3] European Photovoltaic Industry Association (EPIA). Global market outlook for photovoltaics 2013-2017, May 2013.

[4] M. B. Garman. Market microstructure. J. Financ. Econ., 3:257-275, 1976.

[5] L. Grüne. Economic receding horizon control without terminal constraints. Automatica, 49:725-734, 2013.

[6] L. Grüne and J. Pannek. Nonlinear Model Predictive Control. Theory and Algorithms. Springer London, 2011.

[7] Y. Guo, M. Pan, Y. Fang, and P. P. Khargonekar. Decentralized coordination of energy utilization for residential households in the smart grid. IEEE Trans. Smart Grid, 4(3):1341-1350, 2013.

[8] C. A. Hill, M. C. Such, D. Chen, J. Gonzalez, and W. M. Grady. Battery energy storage for enabling integration of distributed solar power generation. IEEE Trans. Smart Grid, 3(2):850-857, 2012.

[9] K. M. M. Huq, M. E. Baran, S. Lukic, and O. E. Nare. An energy management system for a community energy storage system. In Proc. IEEE Energy Conversion Congress and Exposition, 2012.

[10] J. M. Maciejowski. Predictive Control with Constraints. Pearson Education Limited, 2002.

[11] N.-K. C. Nair and N. Garimella. Battery energy storage systems: Assessment for small-scale renewable energy integration. Energ. Buildings, 42(11):2124-2130, 2010.

[12] S. Nykamp, M. G. C. Bosman, A. Molderink, J. L. Hurink, and G. J. M. Smit. Value of storage in distribution grids-Competition or cooperation of stakeholders? IEEE Trans. Smart Grid, 4(3):1361-1370, 2013.

[13] S. Nykamp, A. Molderink, J. L. Hurink, and G. J. M. Smit. Storage operation for peak shaving of distributed PV and wind generation. In Proc. IEEE PES Innovative Smart Grid Technologies, 2013.

[14] E. L. Ratnam, S. R. Weller, and C. M. Kellett. An optimization-based approach for assessing the benefits of residential battery storage in conjunction with solar PV. In Proc. 2013 IREP Symp. Bulk Power System Dynamics and Control-IX, Rethymnon, Greece, 2013.

[15] J. B. Rawlings and D. Q. Mayne. Model Predictive Control: Theory and Design. Nob Hill Publishing, 2009.

[16] S. Shao, F. Jahanbakhsh, J. R. Agüero, and L. Xu. Integration of PEVs and PV-DG in power distribution systems using distributed energy storage - Dynamic analyses. In Proc. IEEE PES Innovative Smart Grid Technologies (IGST), 2013.

[17] C. Slamka, B. Skiera, and M. Spann. Prediction market performance and market liquidity: A comparison of automated market makers. IEEE Trans. Eng. Manag., 60(1):169-185, 2013.

[18] D. Tran and A. M. Khambadkone. Energy management for lifetime extension of energy storage system in micro-grid applications. IEEE Trans. Smart Grid, 4(3):1289-1296, 2013. 